\title{
Incorporation of a spatial source distribution and a spatial sensor sensitivity in a laser ultrasound propagation model using a streamlined Huygens' principle
}

\author{
Jernej Laloš*, Aleš Babnik, Janez Možina, Tomaž Požar \\ University of Ljubljana, Faculty of Mechanical Engineering, Aškerčeva 6, 1000 Ljubljana, Slovenia
}

\begin{abstract}
The near-field, surface-displacement waveforms in plates are modeled using interwoven concepts of Green's function formalism and streamlined Huygens' principle. Green's functions resemble the building blocks of the sought displacement waveform, superimposed and weighted according to the simplified distribution. The approach incorporates an arbitrary circular spatial source distribution and an arbitrary circular spatial sensitivity in the area probed by the sensor. The displacement histories for uniform, Gaussian and annular normal-force source distributions and the uniform spatial sensor sensitivity are calculated, and the corresponding weight distributions are compared. To demonstrate the applicability of the developed scheme, measurements of laser ultrasound induced solely by the radiation pressure are compared with the calculated waveforms. The ultrasound is induced by laser pulse reflection from the mirror-surface of a glass plate. The measurements show excellent agreement not only with respect to various wave-arrivals but also in the shape of each arrival. Their shape depends on the beam profile of the excitation laser pulse and its corresponding spatial normal-force distribution.
\end{abstract}

Keywords: Green's function, laser ultrasonics, optodynamics, piezoelectric sensor, radiation pressure, wave propagation

\section{Introduction}

Ultrasound-propagation simulations in solids are indispensable in laser ultrasonics $[1,2,3,4,5]$, optodynamics [1,6], optical pump-probe experiments [7, 8], studies and applications of acoustic emission [9], nondestructive testing [10], seismology [11, 12, 13, 14, 15] and in metrology for sensor calibration $[16,17,18]$.

Wave-propagation dynamics is described by a set of differential equations, initial and boundary conditions, which, in order to obtain a waveform solution, can be solved in a number of different ways: analytically, using a finite element method (FEM), a finite difference method (FDM), or Green's function formalism. Although the analytical solution is the most desirable, it is often nearly impossible to derive it due to the intertwined complexity of the wave equations, initial and boundary conditions.

FEM and FDM are valid methods for solving such a set of equations, especially for free-form geometries.

\footnotetext{
${ }^{*}$ Corresponding author.

Email address: jernej.lalos@fs.uni-lj.si (Jernej Laloš)
}

They offer only whole numerical solutions for each specific instance and have to be entirely repeated for even a slight change in the geometry of the problem. Their solutions are bandwidth limited, depending on the density of the modeling mesh. Sharp changes in thus-calculated solutions are often accompanied by residual numerical oscillations that appear as unwanted artifacts in the waveform, bearing no physical meaning [19]. For this reason, the modeling of laser ultrasound often requires a dense mesh in order to distinguish different, sharp wavearrivals. This requires high-frequency solutions, which, in turn, require large computing power to obtain them in a reasonable amount of time.

Green's function formalism, when used appropriately, enables modeling of different wave sources and their combinations without any major change to the model. For example, it is possible to model lightpressure-induced waves [20], thermoelastic waves and ablation-induced waves that are all present in laser ultrasonics $[1,2,4]$. Using this method, it is also possible for a composite waveform solution to be broken down to individual waves where each can be isolated in order to discern its propagation. Once Green's functions have 
been calculated or their band-limited counterparts ob- 95 tained experimentally $[19,21]$, this approach allows for 96 significant changes in source-sensor selection without the need to repeat the entire equation-solving process. In another contrast with the FEM and FDM, by means of a deconvolution [22, 23], Green's function formalism 100 can be used to solve a backward problem - finding an 101 unknown input signal from the known output.

The model, described here, is based on the interwoven concepts of Green's function formalism and the sta- 104 tistically streamlined Huygens' principle. It accounts 105 for the real extents of the spatial source distributions and 106 sensor sensitivity distributions. Huygens' principle has 107 been used in waveform modeling before [24, 25, 26], 108 predominantly to describe wide sources, while not si- 109 multaneously including sensor averaging.

The basic constituents of this computational approach 111 are the direct time- and space-domain Green's functions 112 as opposed to the transformed, temporal- and spatial- 113 frequency-domain Green's functions [27]. To obtain ei- 114 ther of them, the elastodynamic partial differential equa- 115 tion are converted to ordinary differential equations with 116 integral transform techniques. The time domain is trans- 117 formed into the temporal-frequency domain with either 118 the one-sided Laplace or the Fourier transforms while 119 a suitable choice for a radially-symmetric source is the 120 Henkel transform which converts a space domain into a 121 spatial-frequency domain [28, 29].

The most demanding step in obtaining the direct ${ }_{123}$ time- and space-domain Green's functions is the inver- 124 sion from the spatial-frequency domain to the direct 125 space domain. The final results can rarely be given as 126 closed-form expressions [30], thus careful numerical in- 127 tegration avoiding numerous singularities has to be per- 128 formed [31].

The temporal dependence of the source is included in our modeling as a convolution of a time-domain Green's function with a suitable temporal profile of the 130 source. Since a convolution in the time-domain corre- ${ }_{131}$ sponds to the multiplication in frequency-domain, the 132 frequency-domain Green's function [27] can be taken as 133 the starting building block of our construction scheme, 134 thus avoiding one unnecessary step in performing the 135 temporal convolution using a numerical routine based ${ }_{136}$ on the fast Fourier transform. One might be as well ${ }_{137}$ tempted to perform the same operation by including 138 the radially-symmetric spatial distribution already in the ${ }_{139}$ space-frequency domain. Even though this step can be ${ }_{140}$ realized in some specific cases [28, 29], it does not gen- 141 erally simplify the computation but rather makes it even 142 more demanding by possibly introducing additional sin- 143 gularities into the inversion integrals.
Here, we provide a detailed step-by-step procedure. We describe how to construct an area-to-area (AA) waveform model from a much simpler point-to-point (PP) model with gradual overcoming of its limitations by incorporating the real geometric arrangements of both the source and the sensor, by means of a set of statistically weighted Green's functions.

A plate is chosen as it is one of the simplest and most frequently encountered geometric shapes, with practical scientific and engineering applications, while the ultrasound is chosen to be induced by circularly symmetric laser pulses. Out-of-plane displacement waveforms are commonly measured on either of its surfaces therefore, to facilitate their comparison and practical usefulness, the time-domain laser-pulse-induced surface waveforms are simulated.

To demonstrate the flexibility of the method, three distribution combinations and their corresponding weight functions are presented; they all incorporate a uniform sensor sensitivity distribution and a uniform (top-hat) [20], a Gaussian and an annular (ring) $[20,26,32,33]$ spatial source distribution.

Such a model enables a more accurate representation of high-frequency waves with wavelengths shorter than the spatial extent of the source and the sensor, while Green's functions inherently provide a well-understood physical interpretation of the wave propagation theory.

Measurements of the light-pressure-induced ultrasound have been carried out using a piezoelectric sensor and two lasers, one with a uniform and the other with a Gaussian beam profile. To illustrate the viability of the AA model, a comparison of the measurements with the AA model and the simpler PP model is examined and evaluated in detail.

\section{Development of the model}

The main physical outlines of the problem are as follows: the transient source acts on the top surface of a homogeneous and isotropic plane-parallel plate and induces transient mechanical waves that propagate through it, i.e. ultrasound. Its surface waveforms, time histories of surface displacements, are of interest, since they are most commonly measured. The waveforms under consideration are comprised of waves that travel either directly from the source or are reflected, however many times, from the plate's surfaces but, at the same time, are not reflected from its sides. The model is thus intended for use in time-frames in which the plate can be considered infinitely large and in cases in which waveforms are measured outside the impact area, since this is a practical and quite common measuring arrangement. 


\subsection{Existing point-to-point model}

Sound propagation in solids is described by a system of wave equations, their initial and boundary conditions, the solutions of which, for a $\delta$-function input component, are Green's functions $g\left(\boldsymbol{v}_{0}, \boldsymbol{w}_{0}, t\right)$. They can be thought of as material transfer functions, which transform an input signal $f_{\delta}\left(\boldsymbol{v}_{0}, t\right)=\delta\left(\boldsymbol{v}-\boldsymbol{v}_{0}\right) \delta(t)$ at a point $\boldsymbol{v}_{0}$ into an output signal $u_{\delta}\left(\boldsymbol{w}_{0}, t\right)$ at a point $\boldsymbol{w}_{0}$. The geometric arrangement is shown in Fig. 1(a). Green's functions are highly specific, depending on the elastic and geometric properties of each material as well as the relative positions of the impulse input and waveform output locations. It is understood that, while not explicitly stated in the notation, Green's functions depend on the elastic constants of the plate along with its thickness.

Because of the $\delta$-function input condition, the direct physical use of the Green's functions is somewhat limited to such cases where said point-to-point approximation applies. Due to their algebraic linearity and temporal invariability, this limitation can be overcome, in the temporal dimension at least, by performing a time convolution of a point-to-point Green's function $g_{\mathrm{PP}}\left(\boldsymbol{v}_{0}, \boldsymbol{w}_{0}, t\right)$ with a temporal part of the point-source impulse distribution. The latter is assumed to have separable temporal and spatial dependencies and can be written as:

$$
f_{\mathrm{P}}\left(\boldsymbol{v}_{0}, t\right)=J_{0} s(t) \delta\left(\boldsymbol{v}-\boldsymbol{v}_{0}\right),
$$

where $J_{0}$ is the magnitude of the source impulse equal to the linear momentum transfer to the plate at a point $\boldsymbol{v}_{0}$ and $s(t)$ is its normalized temporal distribution. In this point-to-point (PP) model, the displacement waveform at a point $\boldsymbol{w}_{0}$ due to a point-source is obtained:

$$
u_{\mathrm{PP}}\left(\boldsymbol{w}_{0}, t\right)=J_{0} \int_{-\infty}^{\infty} g_{\mathrm{PP}}\left(\boldsymbol{v}_{0}, \boldsymbol{w}_{0}, \tau\right) s(t-\tau) \mathrm{d} \tau .
$$

In reality, however, the source impulse usually does not act on an infinitesimally small point on the surface but on a certain macroscopic area. It is for this reason 172 that the spatial limitation of the PP model must be ad- ${ }_{173}$ dressed further in the expanded mathematical models.

\subsection{Incorporation of the spatial source distribution}

In this model expansion, a statistically streamlined Huygens' principle is used to calculate, at a certain detection point $\boldsymbol{w}_{0}$, a displacement waveform $u_{\mathrm{AP}}\left(\boldsymbol{w}_{0}, t\right)$ that was induced by a macroscopic, circularly symmetric source impulse $f_{\mathrm{A}}(\boldsymbol{v}, t)$ impacting a circular area of random points $v$ with a radius of $r_{\mathrm{F} 0}$ and centered at $\boldsymbol{v}_{0}$. (a)
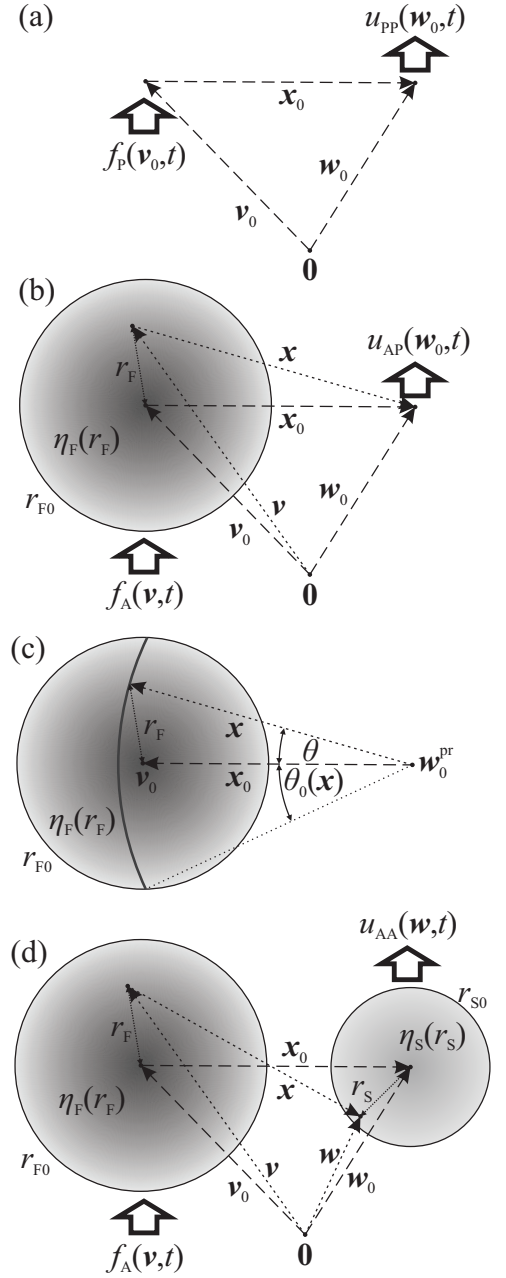

(e)

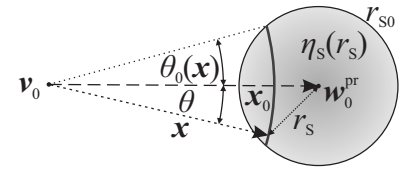

Figure 1: Schematic of coplanar geometric arrangements of different spatial extents (rows) of source (left column) and detection (right column) areas for: (a) PP model, (b) AP model, (c) AP weight function calculation, (d) AA model, (e) PA weight function calculation.

The corresponding geometry is shown in Fig. 1(b). A distinction has to be made between cases where points $v$ and $\boldsymbol{w}_{0}$ are coplanar and where they are not. If point $\boldsymbol{w}_{0}$ does not lie on the impact surface of the plate, as points $\boldsymbol{v}$ by definition do, its projection $\boldsymbol{w}_{0}^{\mathrm{pr}}$ on the impact surface must be used. If those points all lie on the impact surface, however, it is obvious that $\boldsymbol{w}_{0}^{\mathrm{pr}}=\boldsymbol{w}_{0}$.

The real source impulse distribution is represented in the form of:

$$
f_{\mathrm{A}}(\boldsymbol{v}, t)=J_{0} s(t) \eta_{\mathrm{F}}\left(\boldsymbol{v}-\boldsymbol{v}_{0}\right),
$$


where $J_{0}$ is the magnitude of the impulse, $s(t)$ is its normalized temporal distribution and $\eta_{\mathrm{F}}\left(\boldsymbol{v}-\boldsymbol{v}_{0}\right)$ is its normalized circularly symmetric spatial distribution.

A general Huygens' principle states that each perturbed point in space may be regarded as a source of 222 wave propagation. This means that each point in the ${ }_{223}$ source area may be represented as a $\delta$-source weighted ${ }_{224}$ proportionally to the source magnitude at that point in 225 accordance with the overall spatial source distribution. 226 It is therefore quite straightforward to calculate the dis- ${ }_{227}$ placement waveform at a desired point: one simply has 228 to calculate the Green's functions between every point in the source impact area and the point in question and then superimpose them, properly weighted, to obtain the final displacement waveform. Such a straightforward method, however, is not practical as the number of points in an area is, in principle, infinite and the numerical calculation of even a single Green's function is rather demanding and time-consuming.

With the aim of reducing the vast number of Green's functions needed and streamlining the calculations into a more manageable model, a weighted statistical distribution approach is formulated. With it, the twodimensional problem of calculating the weights between random pairs of points and combining them is streamlined to a one-dimensional problem of cumulative weights of pairs of points with equal distance between each-other.

In such an example, where the physical properties of the medium do not change, it makes a significant difference in Green's functions whether the detection point $w_{0}$ lies on the impact surface of the plate or on the opposite surface. Since the detection point $\boldsymbol{w}_{0}$ remains in the same plane throughout the modeling of the waveform, the Green's functions vary only with the relative positions of the input and output points: even less, they vary only with the in-plane distance $x \equiv\left\|\boldsymbol{v}-\boldsymbol{w}_{0}^{\mathrm{pr}}\right\|$ between the two points. It is worth noting that $x_{\min } \leq$ $x \leq x_{\max }$, where $x_{\min }=x_{0}-r_{\mathrm{F} 0}, x_{\max }=x_{0}+r_{\mathrm{F} 0}$ and $x_{0} \equiv\left\|v_{0}-\boldsymbol{w}_{0}^{\mathrm{pr}}\right\|$. Thus, the pairs of input and output points that have the same in-plane distance $x$ between them also have the same Green's function, hereafter in shorthand as $g_{\mathrm{PP}}(x, t)$.

Therefore, instead of performing separate calculations of Green's functions for each pair of points from $v$ to $\boldsymbol{w}_{0}$, it suffices to calculate a normalized weight function $\psi_{\mathrm{AP}}(x)$ for the field of appropriate Green's functions $g(x, t)$ with which the area-to-point (AP) wave- form transfer function is obtained:

$$
g_{\mathrm{AP}}\left(\boldsymbol{v}, \boldsymbol{w}_{0}, t\right)=\int_{x_{\min }}^{x_{\max }} \psi_{\mathrm{AP}}(x) g(x, t) \mathrm{d} x .
$$

The weight function is in essence the weighted distribution of distances between the pairs of points and can be derived with the use of geometrical probability. From Fig. 1(c) it becomes clear how this is done. It has to be emphasized that this model is intended for the use in instances in which the detection point projection $\boldsymbol{w}_{0}^{\mathrm{pr}}$ lies outside the source impact area: $r_{\mathrm{F} 0}<x_{0}$.

At a certain distance $x$ from the point $\boldsymbol{w}_{0}^{\mathrm{pr}}$, corresponding to the appropriate Green's function for the same distance, the spatial source distribution $\eta_{\mathrm{F}}\left(r_{\mathrm{F}}\right)$ has to be integrated in infinitesimally small increments $x \mathrm{~d} \theta$ along the infinitesimally thin arc with the radius of $x$ inside the source impact area. Since the spatial distribution is circularly symmetric, it only changes with its radial component so it can be written in such a simplified form, where $r_{\mathrm{F}} \equiv\left\|\boldsymbol{v}-\boldsymbol{v}_{0}\right\|$. The limits of the integration are at the angles $\theta_{0}(x)$ which represent the points at which the arc with the radius of $x$ and the circular edge of the source area intersect. The normalized weight function is thus:

$$
\psi_{\mathrm{AP}}(x)=\psi_{\mathrm{AP} 0} \int_{-\theta_{0}(x)}^{\theta_{0}(x)} \eta_{\mathrm{F}}\left(r_{\mathrm{F}}(x, \theta)\right) x \mathrm{~d} \theta,
$$

where $\psi_{\mathrm{APO}}$ is the normalization constant.

With the use of the law of cosines, the radial component of the spatial distribution is expressed in terms of $x$ and $\theta$ as:

$$
r_{\mathrm{F}}(x, \theta)=\sqrt{x_{0}^{2}+x^{2}-2 x_{0} x \cos (\theta)},
$$

from which, for $r_{\mathrm{F}}\left(x, \theta_{0}\right)=r_{\mathrm{F} 0}$, the integration limit in Eq. (5) is expressed in terms of $x$ as well:

$$
\theta_{0}(x)=\arccos \left(\frac{x_{0}^{2}-r_{\mathrm{F} 0}^{2}+x^{2}}{2 x_{0} x}\right) .
$$

230 Both expressions are to be inserted in Eq. (5).

Finally, the displacement waveform at the detection point $\boldsymbol{w}_{0}$ induced by the spatial source with a macroscopic impact area is obtained, similarly as in Eq. (2), with a temporal convolution of the rest of the spatial source distribution and the area-to-point waveform transfer function:

$$
u_{\mathrm{AP}}\left(\boldsymbol{w}_{0}, t\right)=J_{0} \int_{-\infty}^{\infty} g_{\mathrm{AP}}\left(\boldsymbol{v}, \boldsymbol{w}_{0}, \tau\right) s(t-\tau) \mathrm{d} \tau .
$$




\subsection{Incorporation of the spatial sensor sensitivity}

Displacement waveforms are measured with sensors which are not infinitesimally small. They are usually able to measure displacements in some macroscopic area of points $\boldsymbol{w}$, which is often circular in shape, with a radius of $r_{\mathrm{S} 0}$ and centered at $\boldsymbol{w}_{0}$. Their sensitivity distribution of $\eta_{\mathrm{S}}\left(\boldsymbol{w}-\boldsymbol{w}_{0}\right)$ is also often circularly symmetric. This means that sensors tend to average out the ${ }_{273}$ measured waveform over the probed area according to 274 its sensitivity distribution, known as the aperture effect. 275 For example, a piezoelectric sensor may have a uniform ${ }_{276}$ sensitivity in its probing area while an interferometer ${ }_{277}$ may use a Gaussian beam with the same probe averag- ${ }_{278}$ ing distribution [34]. For this reason, it is convenient ${ }_{27}$ to develop the model even further and derive an areato-area (AA) transfer function for a displacement waveform. The geometric arrangement in this expanded, AA model is presented in Fig. 1(d).

Similarly as in Sect. 2.2, particularly Eq. (4), the area-to-area waveform transfer function is obtained ${ }_{285}$ by multiplying the normalized weight function $\psi_{\mathrm{AA}}(x){ }_{286}$ with the field of appropriate Green's functions $g(x, t)$ :

$$
g_{\mathrm{AA}}(\boldsymbol{v}, \boldsymbol{w}, t)=\int_{x_{\min }}^{x_{\max }} \psi_{\mathrm{AA}}(x) g(x, t) \mathrm{d} x .
$$

In-plane distance simplifications of $x_{0} \equiv\left\|\boldsymbol{v}_{0}-\boldsymbol{w}_{0}^{\mathrm{pr}}\right\|_{293}^{292}$ and $x \equiv\left\|\boldsymbol{v}-\boldsymbol{w}^{\mathrm{pr}}\right\|$ are also made as well. Here, $\boldsymbol{w}^{\mathrm{pr}}{ }_{294}$ represents the projection of points $\boldsymbol{w}$ onto the impact ${ }_{295}^{294}$ surface, on which points $v$ lie.

It has to be emphasized again that this expanded ${ }_{297}$ model is intended for use in instances where the source ${ }_{298}$ impact area and the sensor probing area do not overlap ${ }_{299}$ in any way: $r_{\mathrm{F} 0}+r_{\mathrm{S} 0}<x_{0}$.

The calculation of the expanded weight function ${ }_{301}$ $\psi_{\mathrm{AA}}(x)$ is done in three steps.

The first step is the same as the one in Sect. 2.2 where ${ }_{303}$ the weighted distribution of distances $\psi_{\mathrm{AP}}(x)$ between ${ }_{304}$ the pairs of points of $\boldsymbol{w}_{0}^{\mathrm{pr}}$ and $\boldsymbol{v}$ is calculated, as seen in ${ }_{305}$ Fig. 1(c).

The second step is quite similar to the first one in its 307 form but the geometry is reversed, as depicted in Fig. 308 1(e), so that a point-to-area (PA) weighted distribution 309 is formed. In it, $\boldsymbol{w}_{0}^{\mathrm{pr}}$ is replaced with $\boldsymbol{v}_{0}$ and $\boldsymbol{v}$ with ${ }_{310}$ $\boldsymbol{w}^{\mathrm{pr}}$, further, $r_{\mathrm{F}}$ is replaced with $r_{\mathrm{S}} \equiv\left\|\boldsymbol{w}-\boldsymbol{w}_{0}\right\|, r_{\mathrm{F} 0}$ with ${ }_{311}$ $r_{\mathrm{S} 0}, \eta_{\mathrm{F}}\left(r_{\mathrm{F}}\right)$ with $\eta_{\mathrm{S}}\left(r_{\mathrm{S}}\right)$, and $\psi_{\mathrm{AP} 0}$ with $\psi_{\mathrm{PA} 0}$, so that the ${ }_{312}$ weighted distribution of distances $\psi_{\mathrm{PA}}(x)$ between the ${ }_{313}$ pairs of points of $\boldsymbol{v}_{0}$ and $\boldsymbol{w}^{\mathrm{pr}}$ is calculated.

The third step combines the two weighted distribu- 315 tions from the first two steps into an expanded weight ${ }_{316}$ function using the spatial convolution:

$$
\psi_{\mathrm{AA}}(x)=\int_{x_{\min }}^{x_{\max }} \psi_{\mathrm{AP}}(y) \psi_{\mathrm{PA}}(x-y) \mathrm{d} y,
$$

which is, of course, normalized. Such an expanded four-dimensional problem is thus streamlined to a onedimensional problem. It should be emphasized that, with convolution, the domain of the expanded weight function $\psi_{\mathrm{AA}}(x)$ also expands so that: $x_{\min } \leq x \leq x_{\max }$, where $x_{\min }=x_{0}-r_{\mathrm{F} 0}-r_{\mathrm{S} 0}$ and $x_{\max }=x_{0}+r_{\mathrm{F} 0}+r_{\mathrm{S} 0}$.

The expression in Eq. 10 can be evaluated quickly using an effective numerical routine based on the fast Fourier transform.

Such a use of spatial convolution can be understood on two levels.

On one level, it can be understood purely statistically. The two simpler weight functions $\psi_{\mathrm{AP}}\left(\left\|\boldsymbol{v}-\boldsymbol{w}_{0}^{\mathrm{pr}}\right\|\right)$ and $\psi_{\mathrm{PA}}\left(\left\|\boldsymbol{v}_{0}-\boldsymbol{w}^{\mathrm{pr}}\right\|\right)$ can be thought of as weighted probability distributions, in which $\boldsymbol{v}$ and $\boldsymbol{w}^{\mathrm{pr}}$ are random variables independent from each-other. To combine them both into another weighted probability distribution $\psi_{\mathrm{AA}}\left(\left\|\boldsymbol{v}-\boldsymbol{w}^{\mathrm{pr}}\right\|\right)$, their convolution has to be performed in accordance with the probability theory [35].

On another level, it can be understood geometrically. In an area-to-point geometry, all points $v$ in the source area that are equidistant, $x$ away from the detection point projection $\boldsymbol{w}_{0}^{\mathrm{pr}}$, lie on an arc. The same is true when the positions are reversed, in a point-to-area geometry. When searching for equidistant points in an area-to-area geometry, however, there are numerous different combination of points that are $x$ away from eachother. Obviously, there are exceptions in the two extreme cases in which there is only one such combination for each of them.

Since the task is to combine the weights of all different pairs of points that are $x$ away from each-other, the opportune operation to choose is convolution. This is because the convolution is an operation on two functions that effectively takes a mirror image of one function, slides it over the other and computes the area under the two functions. It thus combines the two functions into another expanded function by multiplying the values of one function at every $x$ with the values of the other at every $x$ and adding them all at the appropriate $x$ in the new function.

This means that for all different combinations of points, where each point in a pair is from a different area and the distance between them is $x$, the weights from the two weight functions $\psi_{\mathrm{AP}}(x)$ and $\psi_{\mathrm{PA}}(x)$ are properly multiplied and added together to form the ex- 
panded weight function $\psi_{\mathrm{AA}}(x)$ - for each distance $x{ }_{335}$ between points.

Similarly as in Eq. (8) in Sect. 2.2, a sensor displace- 337 ment is obtained by performing a temporal convolution 338 of the rest of the source distribution with the area-to- 339 area transfer function:

$$
u_{\mathrm{AA}}(\boldsymbol{w}, t)=J_{0} \int_{-\infty}^{\infty} g_{\mathrm{AA}}(\boldsymbol{v}, \boldsymbol{w}, \tau) s(t-\tau) \mathrm{d} \tau
$$

\subsection{Discretization}

The integrals from the previous sections should be solved either analytically or numerically without much difficulty. The numerical calculation of an individual Green's function, however, is time consuming and the calculation of many of them even more so. Since the individual Green's functions, by definition, only apply between two points, it is therefore practical to discretize the distance $x$ between points from the two areas to $x_{i}$. In this manner, Eq. (9) for the area-to-area geometry must be discretized as well. Thus the plate transfer function is calculated by superimposing the optimum number of appropriately weighted Green's functions:

$$
g_{\mathrm{AA}}(\boldsymbol{v}, \boldsymbol{w}, t)=\sum_{i} \Psi_{\mathrm{AAi}} g\left(x_{i}, t\right) .
$$

The weights for individual Green's functions are calculated by integrating sections of the continuous weight function:

$$
\Psi_{\mathrm{AAi}}=\int_{x_{i}-\delta x / 2}^{x_{i}+\delta x / 2} \psi_{\mathrm{AA}}(x) \mathrm{d} x,
$$

with the limits set in the middle of the two neighboring discrete distances: $\delta x=x_{i+1}-x_{i}$.

A similar procedure may be done in the area-to-point ${ }_{348}$ and the point-to-area geometries as well.

\section{Specific cases of the weight function}

To this point, the waveform modeling method was described in general terms. For illustrative purposes, three specific weight functions are introduced here, two of which are used in displacement waveform modeling to be compared with measurements further on.

In the case of a laser pulse reflection from the plate's mirror-surface in which only light pressure induces mechanical waves, the ultrasound generating force is normal to the surface and its distribution is directly propor- 353 tional to the laser beam intensity distribution. The most 354 commonly used lasers have a uniform or a Gaussian intensity distribution, while a useful modification of the first is also an annular intensity distribution. Picometerscale surface displacement waveforms are often measured with a piezoelectric contact sensor which is assumed to have a uniform sensitivity distribution under its contact area. An example of each of these distributions and their combined weight functions is presented below.

Since the intermediate objective in this method is to calculate the appropriate weight function for the field of Green's functions, the source force distributions are incorporated in an AA model weight functions along with the sensor's sensitivity under its contact area. The sensor contact area is chosen as circular in shape with a radial component $r_{\mathrm{S}}$, a radius of $r_{\mathrm{S} 0}$ and a uniform sensitivity distribution, which can be written as:

$$
\eta_{\mathrm{S}}\left(r_{\mathrm{S}}\right)= \begin{cases}\eta_{\mathrm{S} 0}, & r_{\mathrm{S}} \leq r_{\mathrm{S} 0} \\ 0, & \text { otherwise }\end{cases}
$$

where $\eta_{\mathrm{S} 0}$ is a normalization constant. This weighted distribution is shown in Fig. 2.

In accordance with the second step of the AA model calculation, the PA weight function is calculated. For this special case, the equivalent of Eq. (5) is reduced to:

$$
\psi_{\mathrm{PA}}(x)=\frac{1}{\pi r_{\mathrm{S} 0}^{2}} 2 x \arccos \left(\frac{x_{0}^{2}-r_{\mathrm{S} 0}^{2}+x^{2}}{2 x_{0} x}\right) .
$$

This PA weight function $\psi_{\mathrm{PA}}$ is shown in Fig. 3 for the case $r_{\mathrm{S} 0}=1$ and $x_{0}=4 r_{\mathrm{S} 0}$.

Note that, in the far-field limit, where $x_{0} \gg r_{\mathrm{S} 0}$, Eq. (15) is further simplified to a distribution:

$$
\psi_{\mathrm{PA}}\left(x \gg r_{\mathrm{S} 0}\right)=\frac{1}{\pi r_{\mathrm{S} 0}^{2}} 2 \sqrt{r_{\mathrm{S} 0}^{2}-\left(x-x_{0}\right)^{2}}
$$

which is symmetric around $x_{0}$.

This part is the same for all of the AA weight functions obtained by convolution with the following three source force distributions.

\subsection{Uniform force distribution}

A uniform or top-hat force distribution is similar to the sensor sensitivity distribution from above. It impacts on a circular area with a radial component $r_{\mathrm{F}}$ and a radius of $r_{\mathrm{F} 0}$, so its spatial distribution can be written as:

$$
\eta_{\mathrm{FUn}}\left(r_{\mathrm{F}}\right)= \begin{cases}\eta_{\mathrm{F} 0}, & r_{\mathrm{F}} \leq r_{\mathrm{F} 0} \\ 0, & \text { otherwise }\end{cases}
$$

where $\eta_{\mathrm{F} 0}$ is a normalization constant. It is depicted in Fig. 2. In accordance with the first step of the AA 


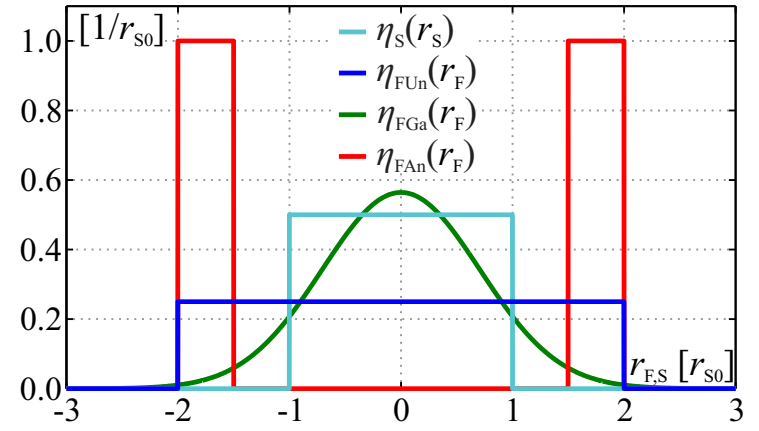

Figure 2: Normalized spatial sensor sensitivity profile $\eta_{\mathrm{S}}\left(r_{\mathrm{S}}\right)$ and three ${ }^{387}$ different source force profiles: uniform $\eta_{\mathrm{FUn}}\left(r_{\mathrm{F}}\right)$, Gaussian $\eta_{\mathrm{FGa}}\left(r_{\mathrm{F}}\right) \quad 388$ and annular $\eta_{\mathrm{FAn}}\left(r_{\mathrm{F}}\right)$

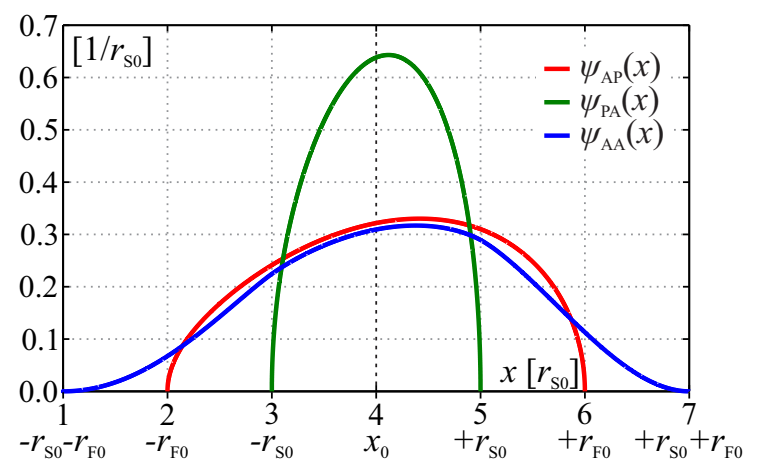

Figure 3: Normalized weight functions in AP, PA, and AA geometries for a uniform source force distribution and a sensor with uniform sensitivity.

model calculation, the AP weight function is calculated.

respective points. Such weight-distance couples were then distributed in a distance histogram, of constant column widths where the height of the interval corresponds to the cumulative weights of all distances that fit in this interval. Such a histogram, of course, containing a large enough number of pairs of points, is a weight function in itself, obtained by the so-called straightforward method. The comparison of the weight functions derived by the two methods for a uniform force distribution is shown in Fig. 4, in which they both match reasonably well.

Interestingly, the area-to-area weight function for uniform distributions in both areas $\psi_{\mathrm{AA}}$ is essentially the same as the distribution of distances between two random points where each is in one of the non-overlapping circular areas. This exact analytical distribution $\psi_{\text {ana }}$ was calculated independently using the theory of geometrical probability [36]. This is also shown in Fig. 4 and it matches the other two methods reasonably well, showing that our AA weighted distribution conforms substantially with both exact distributions.

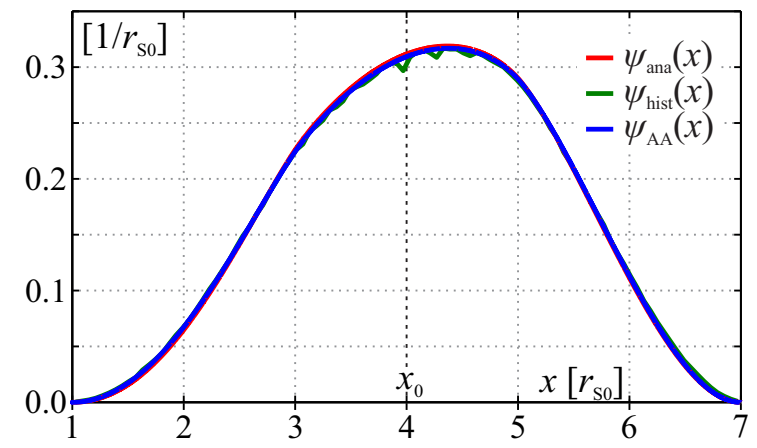

Figure 4: Normalized distributions of distances between points in two non-overlapping circular areas each calculated using a different method: geometrical probability $\psi_{\text {ana }}(x)$ [36], histogram of straightforward method $\psi_{\text {hist }}(x)$ and streamlined Huygens' principle $\psi_{\mathrm{AA}}(x)$.

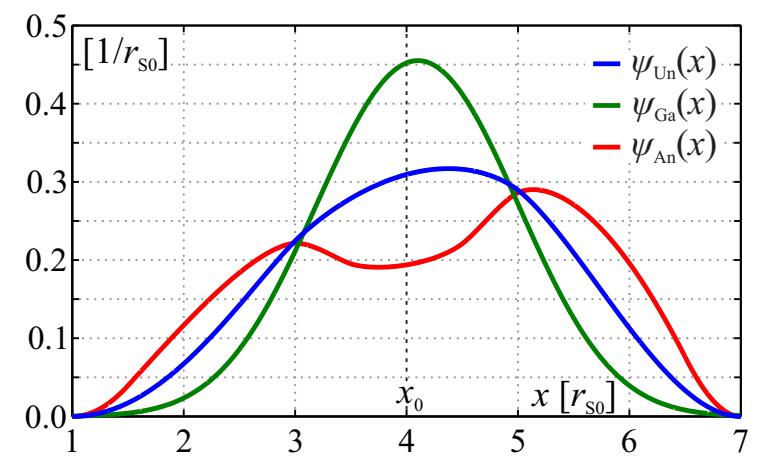

Figure 5: Normalized area-to-area weight functions for a sensor with uniform sensitivity and three different source force spatial distributions: uniform $\psi_{\mathrm{Un}}(x)$, Gaussian $\psi_{\mathrm{Ga}}(x)$ and annular $\psi_{\mathrm{An}}(x)$. 


\subsection{Gaussian force distribution}

A Gaussian force distribution, again presented in Fig. 2 , is produced by a Gaussian beam. The beam is circularly symmetric with a radial component $r_{\mathrm{F}}$ and a beam intensity radius of $r_{\sigma}$, so that its spatial distribution is written as:

$$
\eta_{\mathrm{FGa}}\left(r_{\mathrm{F}}\right)=\eta_{\mathrm{FG}} \exp \left(-2 r_{\mathrm{F}}^{2} / r_{\sigma}^{2}\right)
$$

where $\eta_{\mathrm{FG}}$ is a normalization constant.

The AA weight function $\psi_{\mathrm{Ga}}$ for the Gaussian force distribution is shown in Fig. 5 for $r_{\sigma}=\sqrt{2} r_{\mathrm{S} 0}$.

\subsection{Annular force distribution}

An annular force distribution is similar to the uniform distribution, but it is only constant inside an annulus instead of having a constant value inside a circle. With a radial component $r_{\mathrm{F}}$, an inner radius of $r_{\mathrm{F} 00}$ and an outer radius of $r_{\mathrm{F} 0}$, its spatial distribution can be written as:

$$
\eta_{\mathrm{FAn}}\left(r_{\mathrm{F}}\right)= \begin{cases}\eta_{\mathrm{F} 00}, & r_{\mathrm{F} 00} \leq r_{\mathrm{F}} \leq r_{\mathrm{F} 0}, \\ 0, & \text { otherwise }\end{cases}
$$

where $\eta_{\mathrm{F} 00}$ is a normalization constant. It is depicted in Fig. 2.

The AA weight function $\psi_{\mathrm{An}}$ for the annular force 440 distribution is shown in Fig. 5 for a specifically cho- ${ }^{44}$ sen case of $r_{\mathrm{F} 00}=3 / 4 r_{\mathrm{F} 0}$, where $r_{\mathrm{F} 0}=2 r_{\mathrm{S} 0}$ as in the ${ }_{442}$ uniform distribution example.

All three AA weight functions have also been validated with their respective distance histograms of the ${ }_{445}$ weights of pairs of random points.

\section{Measurements}

In order to demonstrate the viability of this model, 450 two sets of measurements were performed. The wave- 451 forms induced by two different source force distribu- 452 tions were measured on the top surface of a glass plate. ${ }^{453}$ A schematic of the experimental set-up is shown in Fig. ${ }^{454}$ 6.

The plate is made of UV-grade fused silica $\left(\mathrm{SiO}_{2}\right) . \quad{ }^{456}$ It is disk-shaped with a diameter of $d=50 \mathrm{~mm}$ and 457 a thickness of $h=12 \mathrm{~mm}$. Its mechanical properties 458 are: mass density $\rho=2200 \mathrm{~kg} / \mathrm{m}^{3}$, Young's modulus 459 $Y=72 \mathrm{GPa}$ and Poisson's ratio $\mu=0.17$. From these, 460 the following wave propagation velocities are calcu- 461 lated: compressional (P-wave) velocity $c_{\mathrm{P}}=5931 \mathrm{~m} / \mathrm{s}, 462$ shear (S-wave) velocity $c_{\mathrm{S}}=3740 \mathrm{~m} / \mathrm{s}$, Rayleigh $\left(\mathrm{R}-{ }_{463}\right.$ wave) velocity $c_{R}=3387 \mathrm{~m} / \mathrm{s}$. The plate has two coat- 464 ings on its plane-parallel surfaces: a highly reflective 465 (HR) coating on the impact side and an anti-reflective 466

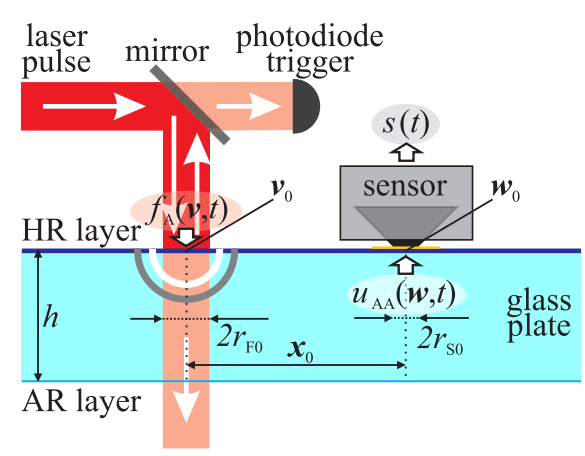

Figure 6: Side-view illustration of the experimental set-up.

(AR) coating on the opposite side. They ensure that the wave-inducing laser light reflects from the top surface as much as possible and that the small amount of light that does pass through the plate reflects from its opposite surface as little as possible, while being absorbed insignificantly either in the coatings or in the substrate. In this way, only the light's momentum transfer to the top surface induces the ultrasonic waves. Conversely, the coatings do not affect the reflection of the laserinduced ultrasound as the thickness of each of them is much smaller than the wavelength of even the shortest detected ultrasonic waves.

Two different laser pulse types with different beam intensity spatial distributions are used to produce different source force distributions for the generation of mechanical waves. Both pulses are produced by Q-switched $\mathrm{Nd}$ :YAG lasers at a wavelength of $\lambda=1064 \mathrm{~nm}$. The first laser pulse (QX MAX, Fotona, Slovenia) is measured to have an energy of $E_{\mathrm{Un}}=200 \mathrm{~mJ}$, a circular impact area with a radius of $r_{\mathrm{F} 0}=1.75 \mathrm{~mm}$ on the plate's surface and a reasonably uniform spatial distribution. The second laser pulse (Brio, Quantel, France) is measured to carry an energy of $E_{\mathrm{Ga}}=55 \mathrm{~mJ}$ and to be circularly symmetric with a Gaussian spatial distribution with a beam radius on the plate's surface of $r_{\sigma}=1.27 \mathrm{~mm}$. Both laser pulses' temporal distributions are measured to have the width in a few nanosecond range. Due to the short duration of the pulses, the duration of both pulses has negligible effect on the calculated waveforms compared to the effects that arise from the spatial distributions. The force impulse $J_{0}$ delivered to the plate is expressed as $J_{0}=2 E / c_{0}$, where $E$ is the pulse energy and $c_{0}$ is the speed of light in vacuum.

The measurements were made with a piezoelectric Glaser-type conical sensor (KRNBB-PC, KRN Services, USA) which is a contact sensor designed to detect out-of-plane displacements on a picometer scale. Its contact area is circular in shape with a radius of 
$r_{\mathrm{S} 0}=0.5 \mathrm{~mm}$ while its sensitivity over the sensing area is assumed to be uniform. The sensor has been calibrated and its transfer function determined, for lower frequencies, in [17] and, for higher frequencies, in [18]. In these, the sensor transfer function in the frequency range $10 \mathrm{kHz}-5 \mathrm{MHz}$ has been determined to be reasonably flat. A thin gold foil was inserted between the sensor and the transparent couplant in order to minimize the light absorption by the sensor's piezoelectric element.

The general geometric arrangement during the measurements was similar to that shown in Fig. 1(d). The center of the sensor contact area was deployed $x_{0}=$ $13.0 \mathrm{~mm}$ away from the center of the laser pulse impact area for both pulse-types. They are arranged in such a way on the plate's surface as to necessitate a maximum travel time for the first significant side-reflected wave to reach the sensor, which is somewhat over $9.0 \mu \mathrm{s}$.

The read-outs of the sensor response signals presented in Figs. 7 and 8 are averaged out from 200 individual repetition for each of the laser pulse types. This is done to reduce the stochastic noise and to improve the signal-to-noise ratio. Each measurement is triggered by a fast photodiode with response time of $1 \mathrm{~ns}$ which detects a small portion of the incident light. Each individual measurement lasts $t_{\Omega}=9.0 \mu$ s while its sampling period is $\delta t=2 \mathrm{~ns}$.

Since the lower frequencies are sparsely represented in both sensor calibrations, due to the limited extent of the measurements, the results of any deconvolution to obtain the time-domain input waveform is coarse. For this reason and since the sensor transfer function is reasonably flat, the deconvolution of measurements does 517 not yield any new information about the input wave- 518 forms. Therefore, the actual output signal measure- 519 ments are presented instead, since they do not deviate 520 significantly from the input waveforms.

In Figs. 7 and 8, the measurements are compared 522 to the corresponding waveform simulations in the pi- ${ }_{523}$ cometer scale calculated using the AA model. Here, the ${ }^{524}$ specific Green's function for a plane-parallel plate have ${ }_{525}$ been calculated by using a modified numerical algo- 526 rithm, initially developed by Hsu [37], which employs ${ }_{527}$ the Cagniard generalized ray method.

\section{Discussion}

The method based on the streamlined Huygens' prin- ${ }_{532}$ ciple provides exact AP and PA weight functions and an ${ }_{533}$ excellent approximation to the exact AA weight func- ${ }_{534}$ tion. When combined with the Green's function for- 535 malism, the resulting waveform simulations provide the ${ }_{536}$

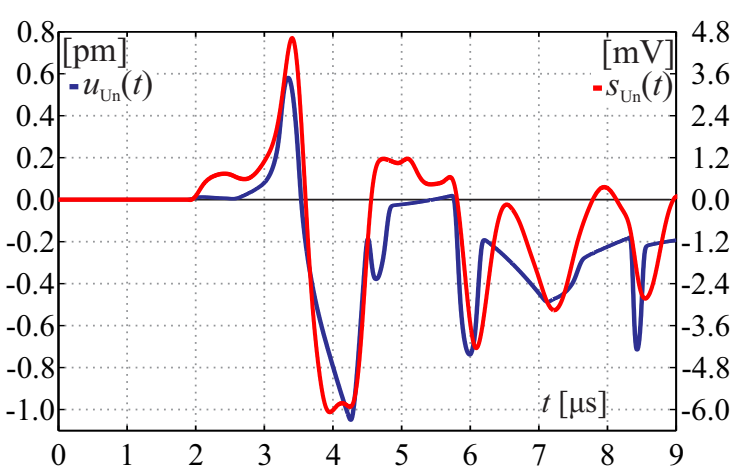

Figure 7: Comparison of the AA model $u_{\mathrm{Un}}(t)$ with the measurements $s_{\mathrm{Un}}(t)$ of light-pressure-induced ultrasound for a uniform source force distribution and a uniform sensor sensitivity with a center-to-center distance of $x_{0}=13.0 \mathrm{~mm}$.

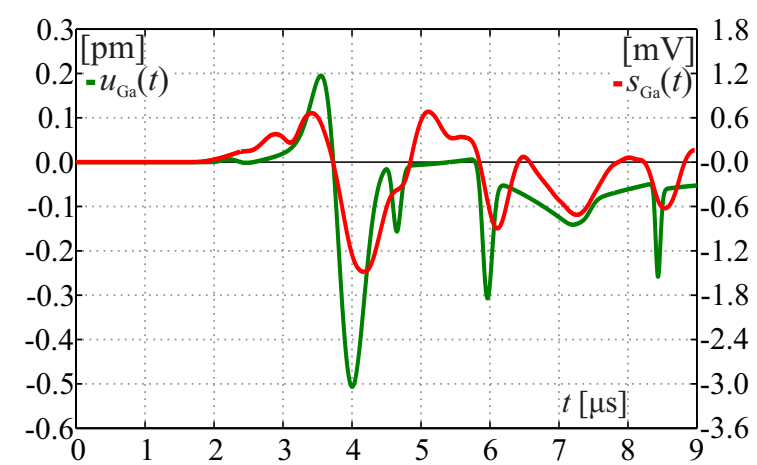

Figure 8: Comparison of the AA model $u_{\mathrm{Ga}}(t)$ with the measurements $s_{\mathrm{Ga}}(t)$ of light-pressure-induced ultrasound for a Gaussian source force distribution and a uniform sensor sensitivity with a center-tocenter distance of $x_{0}=13.0 \mathrm{~mm}$.

options to incorporate the spatial distributions of either the source or the sensor sensitivity or both. In comparison with the other waveform modeling methods, this one has a deeper physical substance instead of relying on purely numerical mathematical methods of the FEM and the FDM. This allows for waveform decomposition or deconvolution [22, 23, 24], while requiring less computing power to obtain forward or backward problem results. The results of the presented method can also be used to verify the waveforms obtained by FEM or FDM.

In general, different source types require appropriate types of Green's functions. In this paper, the normal-force-impulse-input-normal-displacementoutput Green's function $g_{33}$ is used primarily, as it corresponds with the out-of-plane measurements of ultrasound induced by light pressure of the laser pulse. To obtain the appropriate Green's functions for this method, any valid method of calculation would do [19], in general, and even their direct measurement [21] would work, in principle. When a similar wave propa- 
gation is detected on the surface of a solid approximated by an infinite half-space, Green's functions corresponding to that geometry must be used; an analytical example of which is provided by Kausel [30].

To illustrate the effects of different weight functions in the AA model, the surface waveforms for source distributions from Sect. 3, with $r_{\mathrm{S} 0}=2.0 \mathrm{~mm}$, are shown in Fig. 9. All the relevant input parameters for the calculation of Green's functions are the same as those given in Sect. 4.

It can be observed from Figs. 3, 4 and 5 that the weight functions (those obtained by convolution even more starkly) are offset from the center-to-center distance $x_{0}$ towards larger values of $x$. Explained with geometrical reasoning, the largest integration arcs from Eq. (5) appear beyond the center-to-center point of the circular areas, as presented by the specific example of Eq. (15). Of these, the $\psi_{\mathrm{Ga}}$ weight function is offset the least, as the Gaussian distribution is the densest at the center, while the $\psi_{\text {An }}$ has two distinctive peaks, of which the right-handed one is larger, in correspondence with the annular distribution. In the far-field limit, however, the integration arcs become, in effect, straight and the offset disappears, as exemplified by Eq. (16). As seen in Fig. 9, the effect of these offsets translates to the AA waveform models so the wave peaks are slightly delayed compared to the PP model and, in the case of $u_{\mathrm{An}}(t)$, they are split in two.

The approximate AA models were compared to the 589 corresponding distance histograms of the weights of 590 pairs of points. The histograms tend to display a de- 591 gree of coarseness, as is seen in the example of the his- 592 togram in Fig. 4. This follows invariably due to the 593 finite size of the distance-intervals by which individual 594 weights are sorted.

To construct a properly smooth waveform, the dis- 596 crete series of weights multiplied by the Green's func- 597 tions must be smooth as well. The result of the insuffi- 598 cient smoothness can be observed in the literature [28]. 599

The physical understanding of the acquired synthetic 600 waveforms from Figs. 7, 8 and 9 can be enhanced with 601 the ray-schematic presented in Fig. 10. The waveforms 602 are comprised of many different types of wave-arrivals 603 with corresponding propagation velocities, each follow- 604 ing its own path from the source area to the sensor prob- 605 ing area where they all combine into the averaged sur- 606 face waveform as observed by the sensor.

The individual wave arrivals are best seen in the PP ${ }_{608}$ waveform model in Fig. 10, where a single Green's 609 function describes the waveform, with their propaga- 610 tion paths from the source point $\boldsymbol{v}_{0}$ towards the detec- 611 tion point $\boldsymbol{w}_{0}$. The first to arrive are three surface- 612

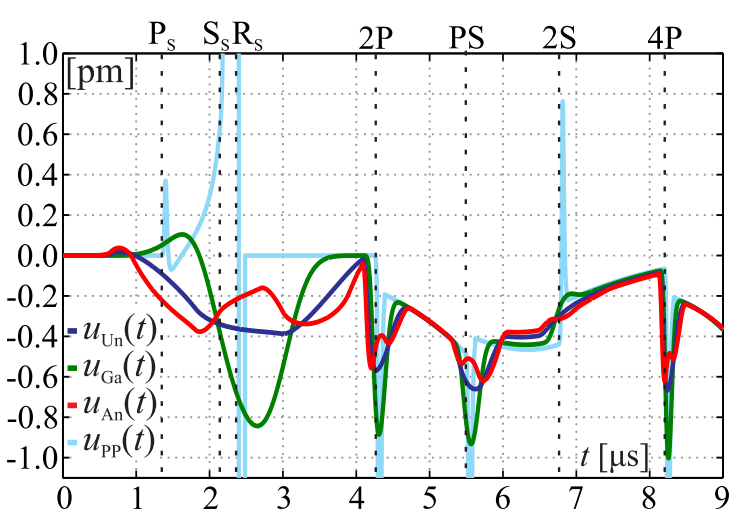

Figure 9: Comparison between the PP model $u_{\mathrm{PP}}(t)$ and the AA model waveforms induced by uniform $u_{\mathrm{Un}}(t)$, Gaussian $u_{\mathrm{Ga}}(t)$ and annular $u_{\text {An }}(t)$ laser pulse profiles from Sect. 3 for $r_{\mathrm{S} 0}=2.0 \mathrm{~mm}$.

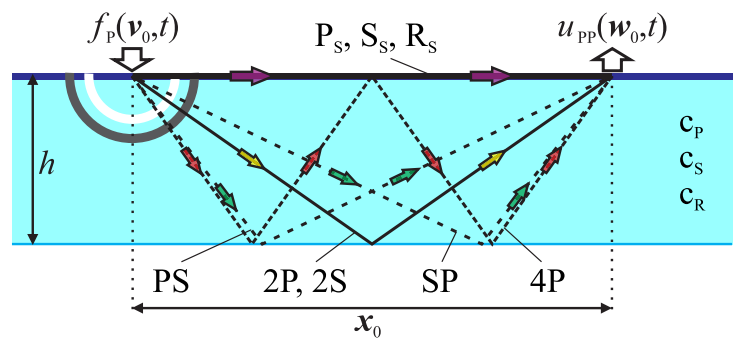

Figure 10: Presentation of propagation paths of different wave types from a point-source to a point-sensor.

propagating waves: the surface-skimming longitudinal wave $\left(\mathrm{P}_{\mathrm{S}}\right.$-wave), seen as a small positive monopolar pulse due to being a compression, the barely noticeable surface-skimming transversal wave $\left(\mathrm{S}_{\mathrm{S}}\right.$-wave $)$, observed as a change in the slope, and the Rayleigh wave ( $\mathrm{R}_{\mathrm{S}}$-wave), with its characteristic dipolar nature carrying the largest amount of energy of all wave types. Up to this point, the waveform is indistinguishable from the half-space surface waveform for the same sourcesensor arrangement and material properties [30]. From this point onward, wave-arrivals are those of indirect bulk-waves that have already been reflected. The once reflected longitudinal wave (2P-wave) is seen as a negative monopolar pulse because the initial compression has been reflected as a rarefaction from the bottom surface. The next wave-arrival is the mode-converted wave (PS-wave) which is a combination of a longitudinal wave being converted to a transversal wave upon reflection (PS-wave) and another wave, arriving simultaneously, where opposite is the case (SP-wave). In the presented time-frame, two more wave-arrivals take place: a once-reflected transversal wave ( $2 \mathrm{~S}$-wave) and a thrice reflected longitudinal wave (4P-wave). In this geometric arrangement, the $x_{0} / h$ ratio does not allow for the 
head waves to appear at the detection point.

The AA model waveform simulations in Fig. 9 dif- 664 fer significantly from the PP model. They have lower 665 amplitudes and wider peaks due to wider spatial distri- 666 butions of the source and wide spatial averaging of the 667 disturbances affecting the sensor. Comparing the AA 668 model waveform simulations of different source force 669 distributions, the differences can be observed most dis- 670 tinctly in the shape of the Rayleigh wave as it is the 671 largest and widest. Of these, the Gaussian source wave- 672 form has the highest amplitudes and resembles the PP 673 model the closest as the source force is concentrated 674 mostly around the center of the impact area, as is con- 675 veyed by its weight function. The distinctive double- 676 peak features of the annular weight function are trans- 677 lated to the waveform in the form of double peaks which 678 are noticeably different from the bell shaped peaks of 679 uniform and Gaussian source waveforms.

Measurements were done using a laser pulse as a light-pressure source [20]. Delivering a very short and consistent force impulse normally to the plate's surface enables a repetition of unvarying stimulations, a property that may not be duplicated using other sources. Thus-induced, picometer-scale surface waveforms were detected by a conical piezoelectric sensor with a limited reliable frequency response with the upper cutoff around $5 \mathrm{MHz}$. The output signal is affected by the sensor's intrinsic transfer function as well as a sort of intrinsic inertia [18] which prevents it to detect very fast changes. This can be observed in Figs. 7 and 8 as slightly altered ${ }^{687}$ and delayed output signals compared to the AA model ${ }^{688}$ input simulations, while making their main features still ${ }^{68}$ discernible.

All of the theoretically predicted wave-arrivals are ${ }^{692}$ also present in the measurements. The difference be- ${ }^{693}$ tween laser pulse intensity distributions is evident when ${ }_{695}^{694}$ comparing Figs. 7 and 8. The most pronounced differ- 696 ence is observed in the Rayleigh wave where its positive 697 and negative polarity peaks from the Gaussian source ${ }^{698}$ are closer together, while from the top-hat source, the ${ }_{700}$ Rayleigh peaks are further apart.

\section{Conclusions}

We have developed a semi-analytical model, based 706 on the streamlined Huygens' Principle, which pre- ${ }^{707}$ dicts high-frequency, out-of-plane surface-displacement ${ }_{709}^{708}$ waveforms in plates for realistic sources and detectors. 710 The wide source of ultrasound was decomposed into an 711 assembly of point radiators, each represented by an ap- ${ }^{712}$ propriate Green's function. The aperture of the sen- ${ }_{714}^{713}$ sor was included in the model as the averaging over 715 the collection of point-waveforms according to the spatially dependent sensitivity. Compared to the finite element/difference methods, our approach excels in the versatility and physical interpretation of the results.

This modeling was used to predict picometer-scale, surface-displacements caused by light-pressure-induced elastic waves when they are measured by a conical PZT sensor. The same model can also be used to describe other, well-established laser ultrasound generating mechanisms, such as ablation or thermoelastic expansion. It can also be used to calculate waveforms generated by mechanical transients (such as: ball drops, radially loaded glass capillary fractures, and pencil lead breaks) or by contact or stand-off transducers (such as: PZTs, capacitive and electromagnetic transducers). Moreover, our approach can as well be employed where the detection area is probed by piezoelectric, electrostatic, electromagnetic or optical means.

\section{Acknowledgements}

The research was conducted as a part of the Optodynamics program (P2-0392), in duration from 20151-1 to 2019-12-31, financed by the Slovenian Research Agency.

\section{References}

[1] T. Požar, A. Babnik, J. Možina, From laser ultrasonics to optical manipulation, Opt. Express 23 (2015) 7978-7990. DOI: 10.1364/OE.23.007978

[2] C.B. Scruby, L.E. Drain, Laser Ultrasonics: Techniques and Applications, Adam Hilger, Bristol [etc.], 1990.

[3] V.E. Gusev, A.A. Karabutov, Laser optoacoustics, American Institute of Physics, New York, 1993.

[4] D. Royer, E. Dieulesaint, Elastic Waves in Solids II: Generation, Acousto-optic Interaction, Applications, Springer-Verlag, Berlin [etc.], 2000.

[5] S.J. Davies, C. Edwards, G.S. Taylor, S. B. Palmer, LaserGenerated Ultrasound - Its Properties, Mechanisms and Multifarious Applications, J. Phys. D Appl. Phys. 26 (1993) 329-348.

[6] J. Možina, R. Hrovatin, Optodynamics - A synthesis of optoacoustics and laser processing, Prog. Nat. Sci. 6 (1996) S709S714

[7] M. Schubert, M. Grossmann, O. Ristow, M. Hettich, A. Bruchhausen, E.C.S. Barretto, E. Scheer, V. Gusev, T. Dekorsy, Spatial-temporally resolved high-frequency surface acoustic waves on silicon investigated by femtosecond spectroscopy, Appl. Phys. Lett. 101 (2012) 013108. DOI: 10.1063/1.4729891

[8] O.B. Wright, Thickness and sound velocity measurement in thin transparent films with laser picosecond acoustics, J. Appl. Phys. 71 (1992) 1617-1629. DOI: 10.1063/1.351218

[9] C.U. Grosse, M. Ohtsu, Acoustic emission testing, SpringerVerlag, Berlin [etc.], 2008.

[10] R.K. Miller, E.v.K. Hill, P.O. Moore, Nondestructive Testing Handbook, vol. 6 - Acoustic Emission Testing, American Society for Nondestructive Testing, Columbus, 2005. 
[11] K. Aki, P. G. Richards, Quantitative Seismology, University Sci- 781 ence Books, Sausalito, 2002.

[12] L.R. Johnson, Green's Function for Lamb's Problem, Geophys. 783 J. R. Astron. Soc. 37 (1974) 99-131. DOI: 10.1111/j.1365- 784 246X.1974.tb02446.x

[13] C.L. Pekeris, The Seismic Surface Pulse, P. Natl. Acad. Sci. 786 USA 41 (1955) 469-480.

[14] G.C. McLaskey, A.M. Thomas, S.D. Glaser, R.M. Nadeau, Fault 788 healing promotes high-frequency earthquakes in laboratory ex- 789 periments and on natural faults, Nature 491 (2012) 101-U114. 790 DOI:10.1038/nature 11512

[15] S.D. Goodfellow, R.P. Young, A laboratory acoustic emission 792 experiment under in situ conditions, Geophys. Res. Lett., 41793 (2014) 34223430. DOI: 10.1002/2014GL059965

[16] ASTM Standard E1106-86(2002)e1, Standard Method for Pri- 795 mary Calibration of Acoustic Emission Sensors, ASTM Inter- 796 national, West Conshohocken.

17] G.C. McLaskey, S.D. Glaser, Acoustic Emission Sensor Cal- 798 ibration for Absolute Source Measurements, J. Nondestruct. 799 Eval. 31 (2012) 157-168. DOI: 10.1007/s10921-012-0131-2 800

[18] J. Laloš, T. Požar, J. Možina, High-frequency calibration of 801 piezoelectric displacement sensors using elastic waves induced 802 by light pressure, Stroj. Vestn.-J. Mech. E. 61 (2015) 533-542. DOI: $10.5545 / \mathrm{sv}$-jme.2015.2731

[19] R.R. Naber, H. Bahai, Analytical and experimental validations of a numerical band-limited Green's function approach for modelling acoustic emission waves, Adv. Eng. Software 38 (2007) 876-885. DOI: 10.1016/j.advengsoft.2006.08.037

[20] T. Požar, J. Možina, Measurement of Elastic Waves Induced by the Reflection of Light, Phys. Rev. Lett. 111 (2013) 185501. DOI: 10.1103/PhysRevLett.111.185501

[21] G.C. McLaskey, D.A. Lockner, B.D. Kilgore, N.M. Beeler, A Robust Calibration Technique for Acoustic Emission Systems Based on Momentum Transfer from a Ball Drop, B. Seismol. Soc. Am. 105 (2015) 257-271. DOI: 10.1785/0120140170

[22] E. Jacquelin, A. Bennani, P. Hamelin, Force reconstruction: analysis and regularization of a deconvolution problem, J. Sound Vib. 265 (2003) 81-107. DOI:10.1016/S0022-460X(02)01441-4

[23] A.S. Carasso, N.N. Hsu, Probe Waveforms and Deconvolution in the Experimental-Determination of Elastic GreensFunctions, SIAM J. Appl. Math. 45 (1985) 369-382. DOI: $10.1137 / 0145021$

[24] C. Chang, W. Sachse, Analysis of Elastic Wave Signals from an Extended Source in a Plate, J. Acoust. Soc. Am. 77 (1985) 1335-1341. DOI: 10.1121/1.392023

[25] H.D. Mair, L. Bresse, D. A. Hutchins, Diffraction Effects of Planar Transducers Using a Numerical Expression for Edge Waves, J. Acoust. Soc. Am 84 (1988) 1517-1525.

26] S. Dixon, T. Harrison, Y. Fan, P.A. Petcher, Thermoelastic laser generated ultrasound using a ring source, J. Phys. D: Appl. Phys. 45 (2012) 175103. DOI: 10.1088/0022-3727/45/17/175103

[27] E. Kausel, Fundamental Solutions in Elastodynamics: A Compendium, Cambridge University Press, Cambridge [etc.], 2011.

[28] L.F. Bresse, D.A. Hutchins, Transient generation of elastic waves in solids by a disk-shaped normal force source, J. Acoust. Soc. Am. 86 (1989) 810-817. DOI: 10.1121/1.398204

[29] L.F. Bresse, D.A. Hutchins, Transient generation by a wide thermoelastic source at a solid surface, J. Appl. Phys. 65 (1989) 1441-1446. DOI: 10.1063/1.342956

[30] E. Kausel, Lamb's problem at its simplest, Proc. R. Soc. A 469 (2013) 20120462. DOI: 10.1098/rspa.2012.0462

[31] A.G. Every, K.Y. Kim, Time domain dynamic response functions of elastically anisotropic solids, J. Acoust. Soc. Am. 95 (1994) 2505-2516. DOI: 10.1121/1.409860

[32] M. Kalms, S. Hellmers, P. Huke, R.B. Bergmann, Beam shap- ing using liquid crystal-on-silicon spatial light modulators for laser ultrasound generation, Opt. Eng. 53 (2014) 044110. DOI: 10.1117/1.OE.53.4.044110

[33] X. Wang, M.G. Littman, J.B. McManus, M. Tadi, Y.S. Kim, A. Askar, H. Rabitz, Focused bulk ultrasonic waves generated by ring-shaped laser illumination and application to flaw detection, J. Appl. Phys. 80 (1996) 4274; DOI: 10.1063/1.363387

[34] T. Požar, J. Možina, Detection of subnanometer ultrasonic displacements, in: K.D. Sattler (Ed.), Fundamentals of Picoscience, CRC Press, Boca Raton [etc.], 2014, p. 553-577.

[35] H. Tijms, Understanding Probability, third ed., Cambridge University Press, Cambridge [etc.], 2012.

[36] A. M. Mathai, An Introduction to Geometrical Probability: Distributional Aspects with Applications, Gordon and Breach, Newark, 1999.

[37] N.N. Hsu, Dynamic Green's functions of an infinite plate - a computer program, Technical Report No. NBSIR 85-3234, National Bureau of Standards, Washington, 1985.

[38] A. McNab, A. Cochran, M.A. Campbell, The calculation of acoustic fields in solids for transient normal surface force sources of arbitrary geometry and apodization, J. Acoust. Soc. Am. 87 (1990) 1455-1465. DOI: 10.1121/1.399442 